\title{
Micro-structural analysis of tablet surface layers by intelligent laser speckle classification (ILSC) technique: An application in the study of both surface defects and subsurface granule structures
}

\begin{abstract}
Purpose : As a consequence of the latest developments in laser technologies it is now possible to develop a low-cost and accurate tablet inspection system by the unification of optical and artificial intelligence methods.

Method: The functionality of the proposed system is based on a sequence of texture analysis of laser speckle images (using laser sources of $650 \mathrm{~nm}$ and $808 \mathrm{~nm}$ : VIS/IR) followed by the optimization of texture parameters using Bayesian Networks (BN). Results: In the first part of this work, a Bayesian inference method was used to detect micro-scale tablet defects that are generated "progressively" during production whereas in the second part a Bayesian classifier method was used to discriminate between tablets made from different granule sizes. In part two, it was shown that (i) the comparatively higher energy $(5 \mathrm{~mW})$ IR laser light generates different speckle effects than the lower energy visible (Red $3 \mathrm{~mW}$ ) by interacting with deeper sub-surface of the tablets and (ii) by using multiclassifier systems (MCS) to fuse the Bayesian classifiers from both types of speckle images it was possible to achieve a higher discrimination power ( $88 \%$ classification accuracy) for distinguishing between tablets made from different granule sizes than one can achieve from a single image type. Conclusion: It is suggested that this unified method has the potential to provide for a comprehensive analysis of both tablet quality attributes, on the one hand, and failure modes, on the other, that might be used in the development of a low cost tablet inspection system.
\end{abstract}

Keywords : Tablet inspection, laser speckle imagery, Bayesian networks, artificial intelligence

\section{Introduction}

Most real-time industrial tablet inspection systems rely on normal visible light sources to characterise basic physical properties, such as the diameter and thickness of the tablet. In another specific example, introduced by GarciaMunoz and Gierer [3], the tablet surface was examined by the use of four fluorescent lights for the inspection of the uniformity of tablet coatings. However, these systems use expensive metrological cameras coupled to highly accurate system configurations and therefore do not present themselves as cost effective systems for widespread industrial use. In addition, these optical systems, are based on non-coherent light sources and are therefore unsuited to the real-time inspection of tablet sub-surface microstructures, for example in the characterization of fluctuations in granule size distributions, micro-density and pore size distributions from variations in the powder blend, the powder grain size, etc. Tracking fluctuations in these parameters would be important in demonstrating the consistency of the product during routine manufacture, and may even go some way to supporting real time release testing. Some studies investigate the use of ultrasonic wave for the real-time tablet inspection [12]. Although the information obtained probes deeper within the tablet surface, this approach has the disadvantage over the optical inspection systems in real-time applications where the tablets under inspection would be in motion and the speed of ultrasound is not as fast that that of light to achieve a pinpoint inspection. Other instrumentation based on coherent light sources (i.e. lasers) has greater potential for probing the sub-surface, e.g. NIR and Raman spectroscopy systems [14][22]. However, the information available is restricted to content uniformity and/or the assessment of tablet density as a key parameter in predicting dissolution rate, for example (rather any specific structural features of the tablet surface and sub-surface).

In last few decades, the rapid developments in low-cost, laser optical technologies and high-tech instrumentation have now made it possible to combine optical and statistical methods in the development of affordable and accurate 
industrial vision systems for tablet inspection. In contrast to the aforementioned methods using visible light, fluorescence, Raman and ultrasound, laser light has the ability to penetrate deep inside a tablet due to its constant wavelength and focused energy. Importantly, and of direct relevance to this work, is the speckle effect which results from the diffuse reflectance and back scattering of laser light, which is specific to tablet micro structure and content.

Laser speckle imaging systems may be implemented by unifying the related image processing methods in association with artificial intelligence tools and low-cost laser-optics instrumentation to reach an optimum accuracy in the classification of tablets as either in- or out-of-specification, with regard to certain attributes associated with the tablet microstructure. Within the proposed system, we acquire laser speckle images from tablet illuminated by lasers at 650 and 808 wavelengths, then apply texture analysis algorithms with 9 texture measures (and then Bayesian classifier or Bayesian inference method). This novel technique is called "Intelligent Laser Speckle Classification (ILSC)" [1][2]. The aim of the current study is to investigate the application of the ILSC technique in the classification of tablets according the surface microstructures associated with different tablet densities from using a range of granule sizes and with the micro-scale defects that result from tablet sticking, with the future possibility of integrating our proposed system with the traditional die-punch set for a low-cost, high speed and accurate tablet inspection process.

\subsection{Tablet Inspection systems}

There are many real-time tablet inspection system providers across the world market. One of them is UK based RNA Systems ${ }^{\mathrm{TM}}$ that provides $360^{\circ}$ tablet inspection of their physical characteristics. The similar one is also Canadian Optel's CountSafe ${ }^{\mathrm{TM}}$ tablet inspection systems providing the almost same testing facilities. Slovenia based company Sensum provides an inspection system SPINE ${ }^{\mathrm{TM}}$ as is capable of precise colour inspection by use of highly specific colour cameras. Such systems above make a real-time checking of the physical tablet characteristics such as size, shape, colour, etc. and also the physical defects on the tablet surface by a real-time sequential image frames acquisition via CCD or CMOS industrial cameras. The systems then perform image processing algorithms such as segmentation (to separate tablet from the background or outline the tablet defect areas), metric measurements on the images, pixel based colour identification, etc. However such systems are unable to examine tablet content or tablet subsurface material characteristics. The reason why they are unable to achieve such tasks is, because such subsurface material characteristics could only be visible to camera or detector (with a proper discrimination) under the illumination of a coherent light (e.g. lasers) or some type of energy sources like THz, ultrasound or x-ray that can penetrate into the tablet subsurface. However, low spatial resolution of $\mathrm{THz}$ and ultrasound energy, and their low discrimination power for very similar tablet materials or their speed of measurements make them unsuitable for content based tablet inspections. Even though there are some experimental scientific (non-real-time) systems introduced by academic studies [24] and one of them utilizes $\mathrm{THz}$ radiation for tablet porosity measurements, however the real-time versions of such systems have not been introduced to the commercial market yet. Only Japanese supplier Ikegami offers a product named TIE-XR ${ }^{\mathrm{TM}}$ which uses $\mathrm{X}$-ray energy for tablet inspection, but there is no indication of tablet content measurement in the system specifications.

The majority of current inspection systems and their techniques rely on normal (non-coherent) light. As far as the physical principles of a normal light energy is concerned, its wavelength has distribution over a broad spectrum unlike a coherent light (e.g. laser) which has always a constant wavelength. Hence the normal light's wavelength composition can easily be changed by its interaction with a tablet, where at the end would be difficult to make an accurate tablet measurement by such inconsistent light form. This causes a difficulty of discrimination between very similar tablet characteristics (e.g. narrow range of density, porosity, etc.) and this is also because of high correlation between the rapid changes on non-coherent light wavelength and those similar tablet characteristics whose information is conveyed by the same interacted light.

\subsection{Physical aspects of laser-surface interactions}

1.2.1 Physical aspects of laser-surface interactions 
The behaviour of laser light reflected from the surface or sub-surface of any object (e.g. a tablet) is highly dependent on the surface characteristics, and can only be modelled by the physical principles of light-material surface interaction. Methods to investigate light-surface interaction phenomenon have been studied by several authors in the past, such as Torrance and Sparrow [15] who introduced a light-surface interaction model, but based on the assumption that the wavelength of incident light is always smaller than the material surface irregularities and therefore the method works only in a limited cases of a number of ideal surface types. However, in the analysis of tablets this is not always be the case as the tablet surface may often be quite 'smooth', with the surface irregularities being presented at the microstructure of individual grains and grain-boundary voids $(0.01-1 \mu \mathrm{m})$ which in turn depend on a number of factors including the powder material used, the compression force applied for compaction, the concentration of lubricant to facilitate release form the punch and die, and any surface coatings and polishing that may be applied.

With another method called the Lambertian bidirectional reflection distribution function, it has already been used for smooth matte-surface reflection modelling [16] but requires perfectly diffuse (matte) surface and ideal illumination conditions which may not be easy to provide for each individual tablet and by noise-free illumination environment within industry.

Nayar and Oren [18] also used a reflectance model for matt surfaces. They state that the effective reflectance from a surface (in a region of interest) varies with magnification and therefore depends very much of the resolution of the imaging sensor. In an industrial environment one would need to ensure that the sensor was positioned close to the tablet under study otherwise image resolution would be adversely affected. Such techniques may therefore be more suited to off-line analysis (with ideal laboratory conditions) rather than in-line.

Tominaga (1991) uses a dichromatic reflection model which recovers the total spectral reflectance from the surface [17] that eliminates the influence of illumination from the use of colour signals. This inevitably leads to lack of discrimination between certain types of tablet surfaces where the colour spectrum of the reflected or transmitted light plays a discrimination role.

Previous studies that were developed specifically for tablet analysis, exploited different locations of energy spectrum with techniques such as $\mathrm{THz}$ pulse energy, time-variant laser speckle, holographic interferometry, etc. Peiponen et al. [25] used THz pulse energy with time delay for tablet measurements. Such scanning based techniques measures only one single point at one time, hence extremely slow for real-time tablet inspection as whole tablet surface scan procedure has to be completed. In addition the calculated spectrum info has to be matched with data base for the final correction procedure. Some of studies used laser speckle but focuses only on tablet deformation during production. Silvennoinen et al. [26] investigate such deformation after the compression but not a comprehensive tablet characterisation. One of the works that uses holographic interferometry [27] exploits inference pattern which is mixture of two different laser speckle patterns produced at different times for diffusion measurements of pharmaceuticals. The disadvantage of such a time-variant technique is, it necessitates a time-variant change in tablet characteristics, which means that if the method is applied onto the homogenous tablet surface, even if the tablet is at continuous speed, it produces two identical patterns with no use.

The ILSC method that we are investigating in this article, having specific surface reflection characteristics, has the potential to overcome the problems experienced in the above reflection models by exploiting the laser speckle phenomenon in association with the supplementary techniques of texture analysis followed by Bayesian methods.

Laser speckle imaging is a well known method [4][10] and is widely used in material surface analysis applications [4], heat transfer analysis [20] and also in fields of bio-imaging [2]. The laser speckle effect is based on a physical phenomenon whereby coherent light (e.g. a laser source) is diffusely reflected and back scattered (diffracted) from a collection of randomly distributed particles to generate a characteristic, random interference pattern which is called the Laser Speckle pattern.

Briers and Webster [11] formulated the laser speckle contrast as: $\quad K=\sigma_{S} /\langle I\rangle$ 
where $\mathrm{K}$ is speckle contrast $(0<\mathrm{K}<1)$ whose ideal value is $1 . \sigma_{\mathrm{s}}$ is the standard deviation of the spatial intensity variations in the speckle pattern $\langle\mathrm{I}\rangle$ and can be taken as the spatial average. The degree of penetration of laser light into a tablet sub-surface will inevitably depend on the intensity (energy) and the wavelength $(\lambda)$ of the source. Figure 1 shows a schematic of how the method can be applied to the assessment of differences in the topography of the tablet and the sub-surface micro-structure of pharmaceutical tablets.

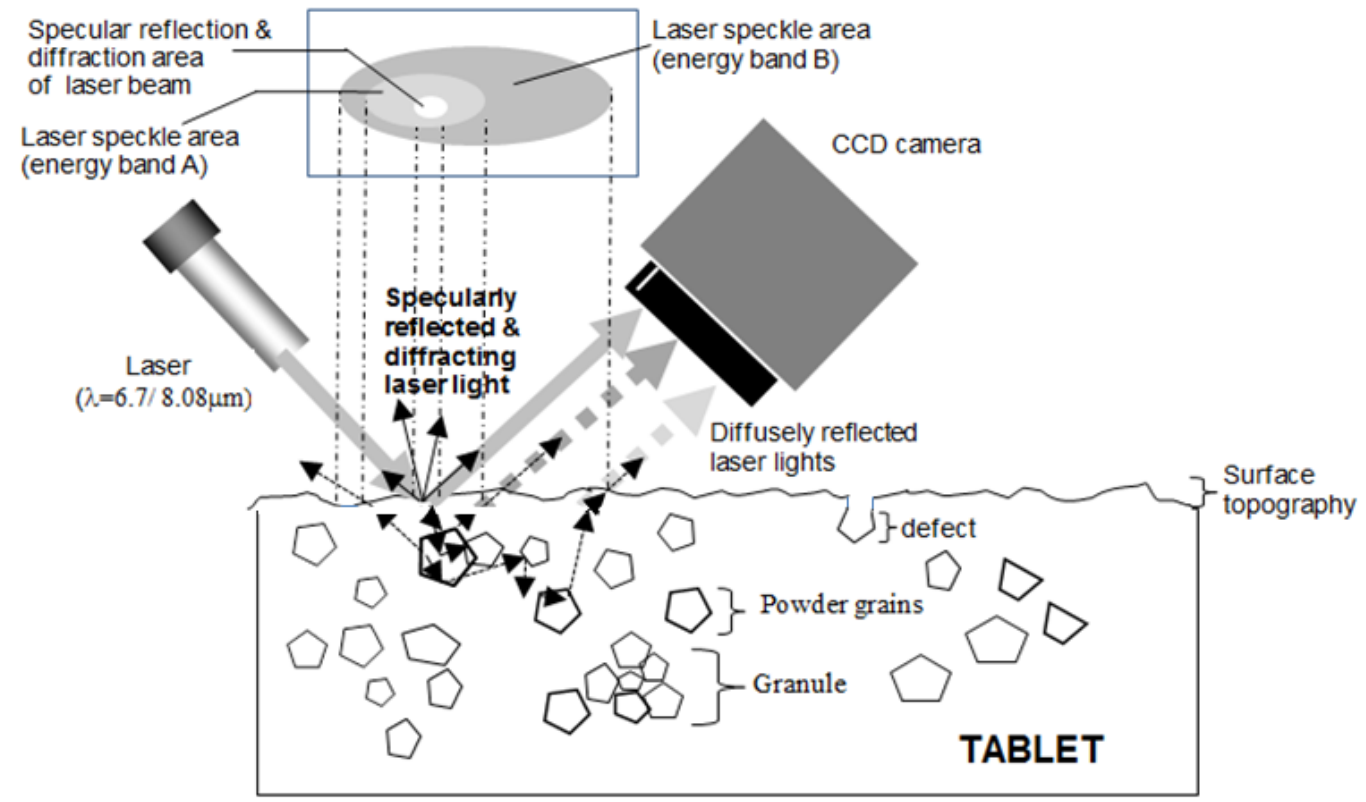

Figure 1. Types of laser light reflection: a) diffraction \& specular reflection b) diffuse reflection and their consequent speckle effect (shown as dotted line arrow and bold line arrow respectively). Here, the diffusely reflected light conveys the sub-structure of tablet to an image domain (i.e. the laser speckle areas, A and B) whereas the specular reflection and diffraction (mixed) area is shown as blank on the image (due to highest laser beam energy) and hence gives no information. The speckle effect is caused by mixture of both reflections from the tablet surface and sub-surface layers, hence creating a tablet-laser interaction in which the components are a function of the particular laser wavelength (at red $\lambda=670 \mathrm{~nm}$ or IR $\lambda=808 \mathrm{~nm}$ ) and the tablet microstructure (shown here as irregular sizes and shapes of granules, powder grains and defects). The camera image displays the laser lightsurface interaction features as also shown in Figure CC.

Pharmaceutical tablets are manufactured from either granules (in the case of wet or dry granulated systems) or from directly compressed blends of powders. The tablet microstructure, which includes the spatial distribution and composition of the various formulation components (the active, the binders, disintegrants, and lubricants, etc.), the grain size distribution of the individual powders and the granule size distribution (if the powder blend has been granule prior to compression), and the pore size distribution (from voids between particles) is a complex function of many material attributes and process parameters, such as the compression characteristics of powder grains and granules, the uniformity of the blend, and the force-compression profile used in the manufacture of the tablet. Collectively, these parameters define the tablet surface topography and the sub-surface structure and are hence responsible for the generation of broad distribution of laser speckle patterns. Moreover, when there are instances of defects being formed on the tablet surface (for example if there is any adhesion/sticking to the punch face, [23]) then these changes in tablet surface topography may also be seen the changes to the laser speckle image. The potential exists therefore for laser speckle methods to be used in the assessment of the tablet quality attributes at various scale lengths from the molecular, to the microscale, mesoscale and macroscale.

The targets for our investigation are (i) the macroscale characteristics associated with the surface topography of the tablet and the impact of tablet sticking thereon, (ii) the microscale characteristics associated with the size of granules used in the preparation of tablets. 


\section{Materials \& Methods}

Two aspects were explored in this study with respect to the discrimination capability of intelligent laser speckle classification (ILSC) technique for certain quality attributes. The first aspect was to research the potential application of $I L S C$ in the detection of surface defects that are a direct result of punch adhesion (i.e. tablet sticking). The second aspect was to assess whether ILSC could be used to differentiate between sub-surface features such as that associated with the granule size.

\subsection{Materials}

In both studies we have used granules of mefenamic acid (Sigma Aldrich, UK) which have been prepared by a wet granulation method from a mixture of $25 \% \mathrm{w} / \mathrm{w}$ mefenamic acid with other excipients (Table 1) that were first blended by shaking in an inflated polythene bag for 1-2 min before granulating in a Procept 4M8 granulator with the agitator blade rotating $1000 \mathrm{rpm}$ and the chopper blade rotating at $3500 \mathrm{rpm}$ while $76 \mathrm{ml}$ of double distilled water was added at a rate of $15 \mathrm{ml} \mathrm{min}^{-1}$. Granulation then continued for a further 3 min and the wet granules then sieved through a range of BS410 stainless steel sieves (of 0.25, 0.5, 1 and $2 \mathrm{~mm}$ mesh size) to produce granules of varying size, which were then dried for one hour at $70 \mathrm{C}$, prior to the addition of $0.5 \%$ magnesium stearate, followed by shaking the granules in a polythene bag for 1-2 min.

Table 1. Powder blend for wet granulation

\begin{tabular}{|l|l|l|l|}
\hline Material & \multicolumn{1}{|c|}{ Supplier } & $\%$ & $200 \mathrm{~g}$ \\
\hline mefenamic acid & Sigma-Aldrich & 25 & $50 \mathrm{~g}$ \\
\hline Lactose monohydrate & Foremost & 49.5 & $99 \mathrm{~g}$ \\
\hline Hydroxyl Propyl Cellulose & Ashland & 20.0 & $40 \mathrm{~g}$ \\
\hline $\begin{array}{l}\text { Avicel PH101 Microcrystalline } \\
\text { cellulose (MCC) }\end{array}$ & FMC Biopolymer & 5.0 & $10 \mathrm{~g}$ \\
\hline
\end{tabular}

a) Surface sticking

In order to investigate the sticking phenomenon, tablets from the $1 \mathrm{~mm}$ mesh size were then prepared on a single punch hydraulic compaction simulator (Phoenix Calibration and Services Ltd, Brierley Hill, UK) using an instrumented adhesion punch with a $10 \mathrm{~mm}$ diameter removable tip (Standard \#1). A single ended sine wave profile with an average punch speed of $300 \mathrm{~mm} / \mathrm{s}$ was used. The resultant tablet weight range was $0.22-0.26 \mathrm{~g}$ with a thickness range of 2.5-3.00 $\pm 0.01 \mathrm{~mm}$. The tablet density range was $9.5-10.5 \mathrm{~kg} / \mathrm{m}^{3}$. A set of microcrystalline cellulose tablets were prepared as a control set given the expectation that this material would not show any sign of sticking.

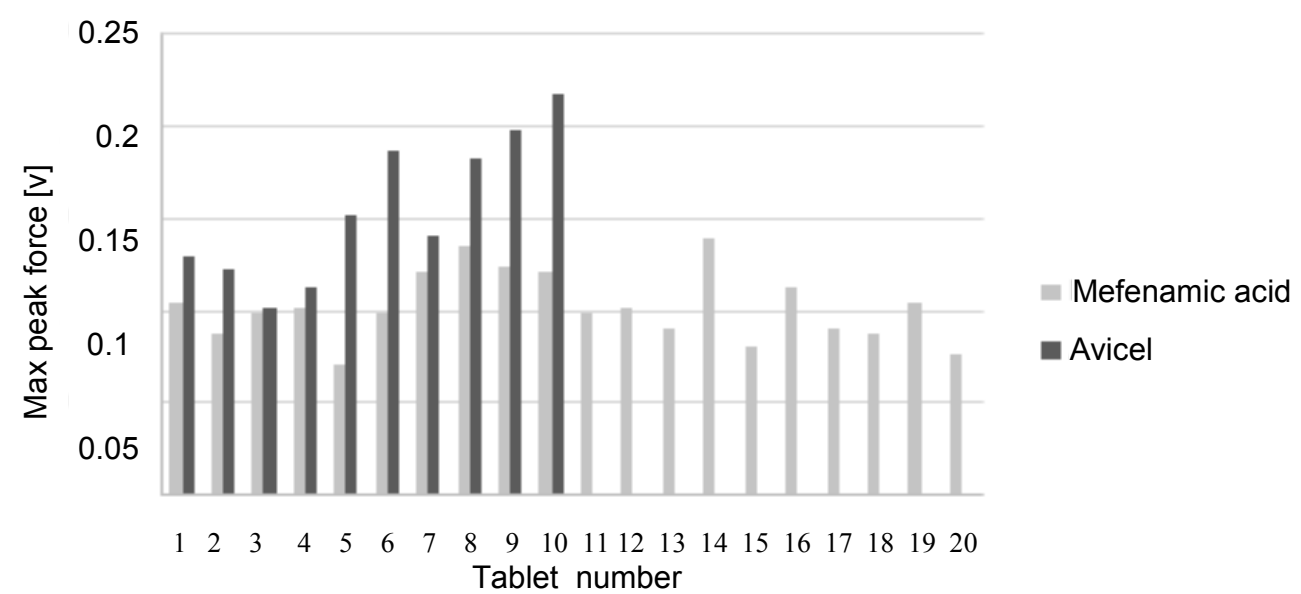

Figure 2. The mean adhesion signal values for (20) mefenamic acid and (10) Avicel PH101 tablets 
The mean adhesion signal (equivalent to the detachment force, Fig. 2) for the mefenamic acid tablets (mean=0.1 and st.deviation $=0.018$ for range $=0.071$ to 0.139 ) was significantly lower than that for the Avicel PH101 tablets (mean $=0.15$ and standard deviation $=0.039$ for range $=0.102$ to 0.217 ) suggesting a reduced tendency for sticking. And yet the visual evidence clearly shows that sticking takes places preferentially with mefenamic acid and not Avicel PH101. The explanation is clear, in that the cohesive forces between the mefenamic acid granules must lower than in the Avicel PH101 powder, such that the adhesive forces between the punch and the mefenamic acid granules are sufficient to cause detachment of material from the tablet compact. Studies of adhesive forces alone are therefore unlikely to provide predictive models for sticking. Our second observation was that there was no apparent trend in the magnitude of the detachment force (either increasing or decreasing with time) which might have otherwise suggested that the forces of attraction between the punch interface and the tablet surface were impacted by an accumulation of powder on the surface of the punch. To qualify that the detachment force did not change over the production cycle, a simple t-test was undertaken with one set taken from the detachment forces of the first six tablets and the second set taken from the last six tablets. The t-test for first 6 and last 6 tablets adhesion signal sets mefenamic acid tablets, gave $\mathrm{t}=0.263<\mathrm{t}$ whereas the critical $=1.81$ indicating that the two sets were indistinguishable in terms of the adhesion force of detachment, at a confidence level of $95 \%$.

A visual examination of the tablets was followed by a scoring of the severity of the defect in order to qualify that there was a progressive, real time, increase in the extent of defect formation as the run of tablets progressed. This scoring was based on $1=$ no defect; 2 = small visible defect; and 3 = major defect as in Figure 3 (top). Fig. 3 (bottom) then shows the time scale for defect creation for the first 20 tablets to be ejected from the compaction simulator.
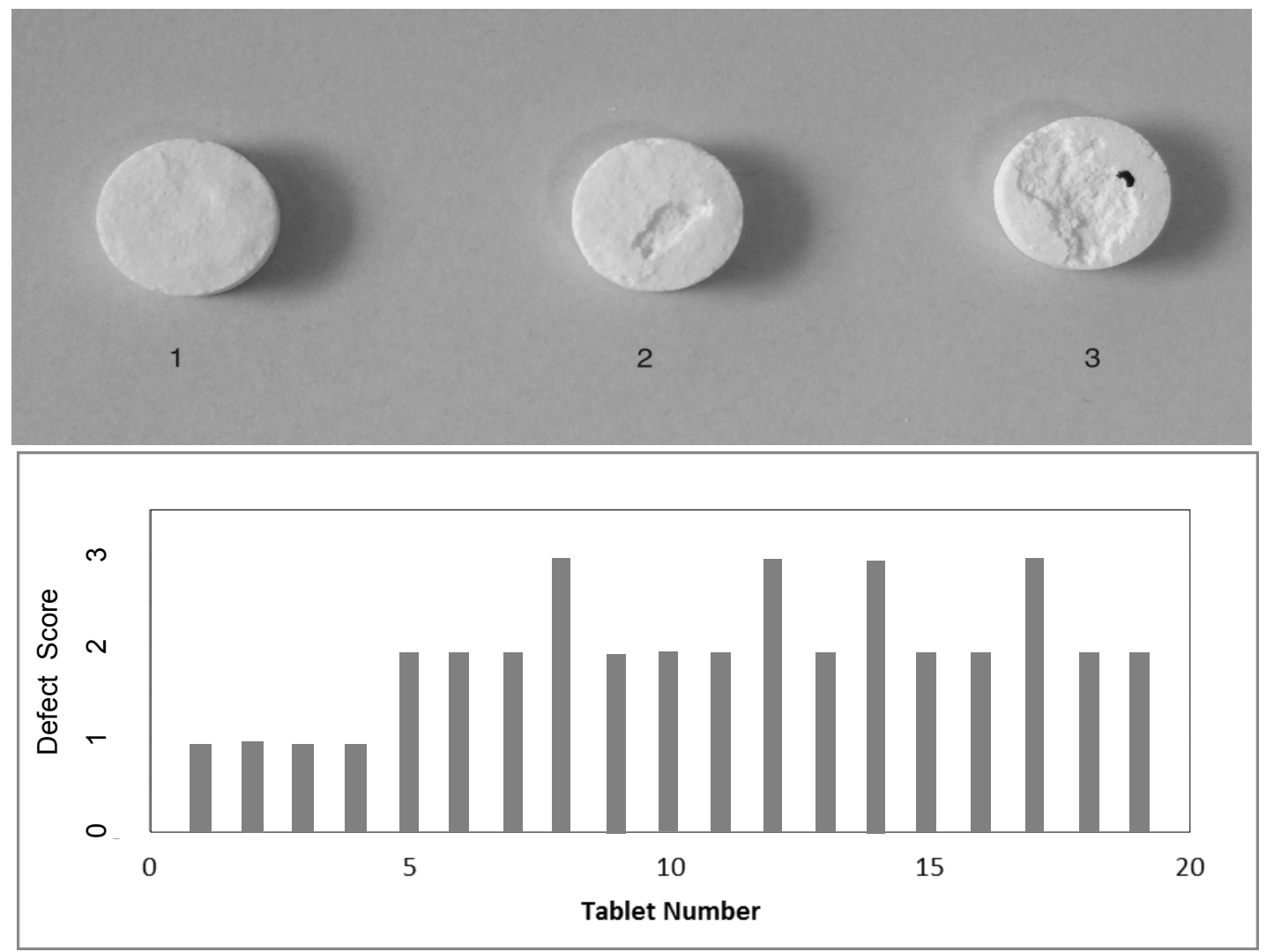

Figure 3. Image of tablets (on the top) showing the scale of defects. Defect score of $1:$ No defects, Defect score of 2 : Small visible defect, Defect score of 3 : Major defect with missing pieces. The graph on the bottom shows the rating of defects on mefenamic acid tablets 


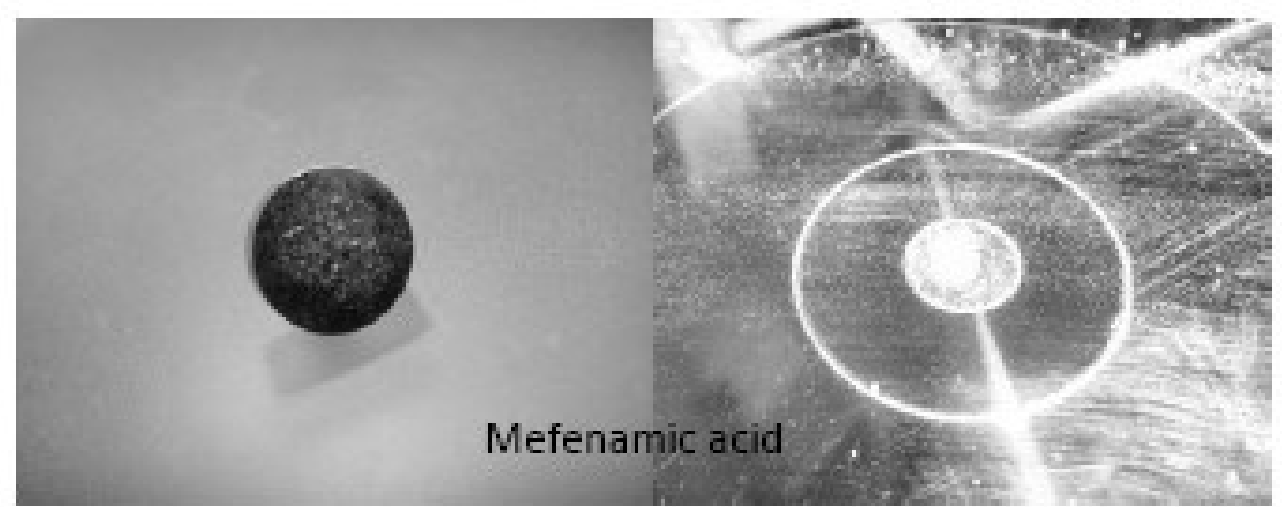

Figure 4. Upper punch tip and lower punch of the compaction simulator showing material deposited on the punch faces as a result of sticking.

Tablet sticking is presumed to result during the tablet production process if the adhesive force exceeds the cohesive force such that the material will then have a propensity to stick to the tablet surface. This phenomenon may develop progressively with time given the increase in the number of opportunities for sticking and the probability that heating of the punches and die increases the likelihood of sticking to occur. However, with our tests this phenomenon occurred very soon after the start of the tablet production run with the $5^{\text {th }}$ tablet showing small defects which then get worse, on average, throughout the production run. There was no visual evidence of sticking with the control set of Avicel® tablets

The sticking test is expected to find a link between the attribute of "time" and the progressive tablet defects developed in time domain (as explained in Section 3.3). However it should be noted that, both the defected area and non-defected area on a tablet among the defected group influenced the classification process in same manner having been different from the non-defected group. This is because for a potentially defected tablet (tends to develop a defect in further stages) even non-defect tablet area characteristics differ from a normal (non-defect) tablet.

b) Sub-surface microstructure: The impact of granule size

To investigate the tablet sub-surface microstructure, granules from the $0.25,0.5$, and $1 \mathrm{~mm}$ mesh size were used for the preparation of tablets with a CT5 single punch machine (Engineering Systems, Nottingham). The $2 \mathrm{~mm}$ granules did not produce tablets of sufficient mechanical strength so were discarded without further consideration. A $10 \mathrm{~mm}$ die and punch was used to produce individual tablets. The filling procedure involved manual transfer of $\sim 250 \mathrm{mg}$ of granules (accurately weighed) to the die and a compaction force of $500 \mathrm{~kg}$ applied at a punch velocity of $3 \mathrm{~mm} / \mathrm{s}$. Each tablet was individually prepared and inspected for defects. Punch faces and die (Figure 4) were cleaned in between each tablet to ensure defect free tablets were produced.

The densities of the tablets from each granule size were assessed having measured the tablet thickness to two decimal places on a digital micrometer and the weight of each on a 4-place analytical balance (see Table 2).

Table 2. Tablet characteristic values for the three sizes of granules

\begin{tabular}{|l|l|l|l|l|}
\hline $\begin{array}{c}\text { Number of } \\
\text { Tablets }\end{array}$ & $\begin{array}{c}\text { Granule size } \\
(\mathbf{m m})\end{array}$ & $\begin{array}{c}\text { Average density } \\
\left(\mathbf{g} / \mathbf{m m}^{\mathbf{3}} \mathbf{)} \pm 1 \mathrm{SD}\right.\end{array}$ & $\begin{array}{c}\text { Average } \\
\text { hardness (N) } \\
\pm 1 \text { SD }\end{array}$ & $\begin{array}{c}\text { Average thickness I } \\
\text { Weight (mg) }\end{array}$ \\
\hline 6 & 1 & $1.20 \pm 0.02$ & $90.63 \pm 19.44$ & $2.5 / 250$ \\
\hline 6 & 0.5 & $1.03 \pm 0.01$ & $23.95 \pm 3.23$ & $3.0 / 250$ \\
\hline 6 & 0.25 & $1.08 \pm 0.01$ & $23.59 \pm 3.23$ & $2.9 / 250$ \\
\hline
\end{tabular}

Statistically significant similarity between each set of tablets was determined by a simple student t-test. It was found that (with $95 \%$ confidence, $\mathrm{t}=11.8>\mathrm{t}$, critical one tail $=1.75$ ) there was statistically significant similarity between 
two sets, i.e. the tablets produced from the $1 \mathrm{~mm}$ granules and a combination set from the tablets produced from both the $0.25 \mathrm{~mm}$ and $0.5 \mathrm{~mm}$ granule sizes. Another student t-test for two categories of $0.25 \mathrm{~mm}$ and $0.5 \mathrm{~mm}$ granule sizes yields the result (with $95 \%$ confidence, $t=12.5>t$, critical one tail $=2$ ) that a statistically significant similarity between two sets (Table 3). The high classification results of the groups (as shown in Table 4) were yielded by ILSC method despite their similarity.

Table 3. In regards to $t$-critical values ( $t$-calculated $<\mathrm{t}$-critical) the granulation density groups appear to be similar

\begin{tabular}{|l|l|l|l|}
\hline $\begin{array}{l}\text { t-test values for } \\
\text { granule sizes }\end{array}$ & $1 \mathrm{~mm}$ & $0.5 \mathrm{~mm}$ & $0.25 \mathrm{~mm}$ \\
\hline $1 \mathrm{~mm}$ & - & 0.2 & 0.00001 \\
\hline $0.5 \mathrm{~mm}$ & - & - & 0.19 \\
\hline $0.25 \mathrm{~mm}$ & - & - & - \\
\hline
\end{tabular}

Table 4 . The classification results indicate that, the granulation density group can be classified at high accuracy despite their similarity

\begin{tabular}{|l|l|l|l|}
\hline $\begin{array}{l}\text { Classification results } \\
\text { for granule sizes }\end{array}$ & $1 \mathrm{~mm}$ & $0.5 \mathrm{~mm}$ & $0.25 \mathrm{~mm}$ \\
\hline $1 \mathrm{~mm}$ & - & $80 \%$ & $83 \%$ \\
\hline $0.5 \mathrm{~mm}$ & - & - & $80 \%$ \\
\hline $0.25 \mathrm{~mm}$ & - & - & - \\
\hline
\end{tabular}

\subsection{Laser Speckle Imaging}

Laser speckle images (each of dimension $1296 \times 964$ pixels) were generated by a $1 / 3$ " progressive scan CCD camera with illumination from an IR laser $(\lambda=808 \mathrm{~nm}, 4.5 \mathrm{~mW})$ and red laser $(\lambda=650 \mathrm{~nm}, 3 \mathrm{~mW})$. The components with the specific system configuration are shown in Figure 5.

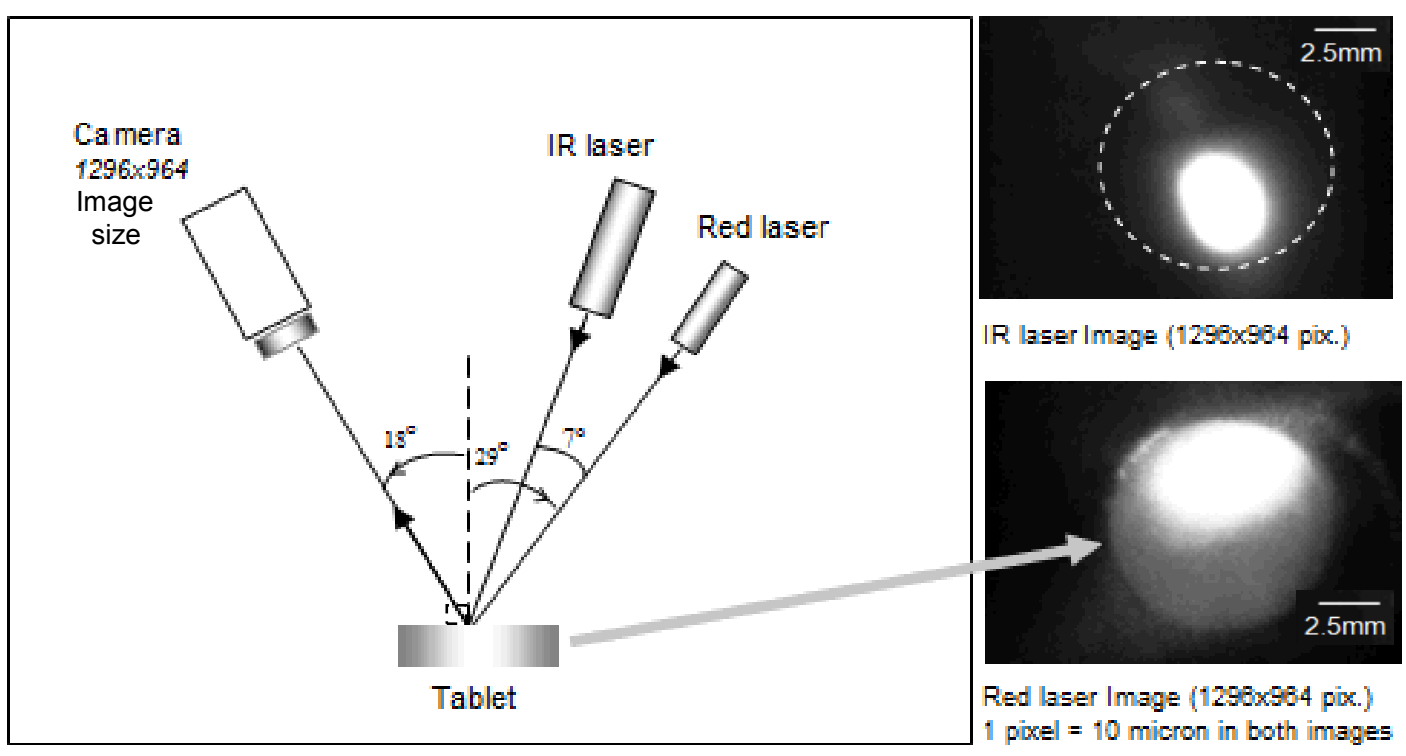

Figure 5. Laser speckle image acquisition setup configuration (on the left) which is a part of complete tablet inspection system and the IR and Red laser speckle images each at 1296x964 pixel size are acquired by the CCD camera which are shown as IR (top-right) and red laser speckle images (bottom-right). In the IR laser image the tablet is not illuminated sufficiently and its location is highlighted by a dotted-lined circle. The IR laser beam width (beam diameter) is $2.5 \mathrm{~mm}$ and the tablet diameter is $10 \mathrm{~mm}$. 
Since the geometric configuration of the system components may vary broadly, it is important to stress that the laser speckle technique is based on comparative analysis rather than absolute measurements. Moreover, the measurement uncertainties of the system from fluctuations in environmental laboratory conditions (temperature and humidity) and camera lens distortion, etc. are common and may be ignored for these comparative measurements, provided the timescale of the fluctuation is long compared with the timescale of the observation window.
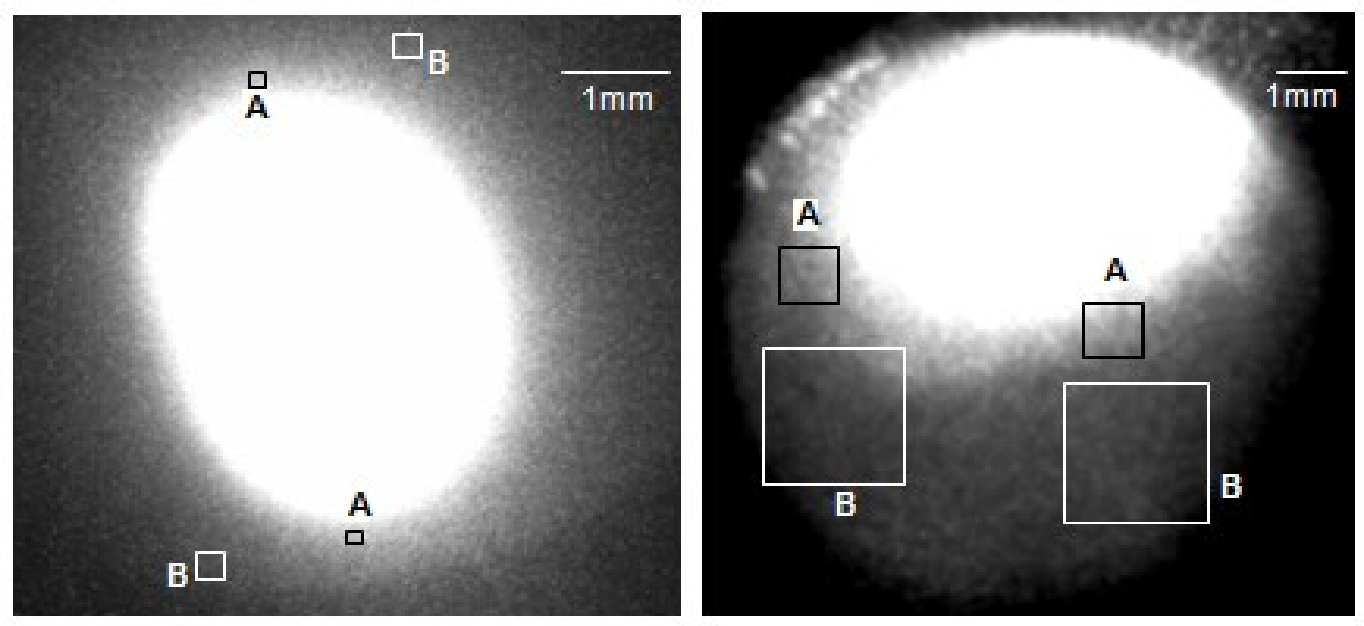

Figure 6. A typical IR(left) and Red (right) Laser speckle images each exhibiting two types of energy bands (high=A; low=B) (hardly visible on black\&white image) on which four sampling regions at different sizes (between $15 \times 15$ and $200 \times 200$ ) are selected. For the sampling procedure, a balance has to be kept between the i) uniformity, ii) max sampling window size within a specific energy band (which places a restriction on the maximum size of the window, in relation to the width of the energy band), and iii) the requirement of sufficient texture information within each the sampling window (which places a minimum of the size of the sampling window). These restrictions have to be considered when selecting the form size and location of the sampling windows in each bands. The highest energy regions are shown as blank which does not contain any speckle information hence not to be used (The images are rescaled with reference to those shown in Figure 5)

\section{a) Texture analysis}

Texture measures shown by Formulas 1-5 were applied to sampling windows of different sizes in pixel unit (e.g. $15 \times 15$ and 30x30 used for $I R$ laser images, $80 \times 80$ or 200x200 used for Red laser images) depending on the relative sizes of the energy bands from each laser source (as shown in Figure 6). In the current configuration, these windows are of approximate size: $(15 \times 15)=0.15 \times 0.15 \mathrm{~mm},(30 \times 30)=0.3 \times 0.3 \mathrm{~mm},(80 \times 80)=0.8 \times 0.8 \mathrm{~mm}$ and $(200 \times 200)$ $=2 \times 2 \mathrm{~mm}$. Up to $4-6$ adequately separated sampling windows were selected across the tablet surface from the regions where the visible laser speckle reflections are effective in terms of sampling uniformity and inclusion of sufficient texture primitives [20]. The independency of cases (tablets surfaces) in the data set is taken into consideration carefully; hence the series of different sampling windows are taken as attributes rather than as cases in the data set. This is because the samplings on the same tablet surface can not be taken as independent cases in a data set. Only the average values of the texture measures from the same size of windows maybe considered as attributes of the data set. 


$$
\begin{aligned}
& \text { Variance }_{\text {Russ }}=\sqrt{\sum(\text { centerpixel }- \text { neighbor })^{2}} \quad \text { (Russ) } \\
& \text { Variance }_{\text {Levine }}=\frac{1}{\text { area }} \sum(\text { centerpixel }- \text { mean })^{2} \quad(\text { Levine }) \\
& \sigma=\sqrt{\text { Variance }_{\text {Levine }}} \\
& \text { Skewness } \left.=\frac{1}{\sigma^{3}} \frac{1}{\text { area }} \sum(\text { centerpixel }- \text { mean })^{3} \quad \text { (Skewness }\right) \\
& \text { Std.Deviation }=\sqrt{\frac{\sum\left(x-x^{\prime}\right)^{2}}{n}}
\end{aligned}
$$

\section{b) Bayesian networks}

A Bayesian network (BN) is a well known artificial intelligence (AI) method which provides a comprehensive data analysis [4][5] particularly for the classification of material surface types in association with laser speckle image data due to its comparative assessment characteristics. The Bayesian package used for this work comprises two different utilities: PowerPredictor ${ }^{\mathrm{TM}}$ and PowerConstructor ${ }^{\mathrm{TM}}$. The first utility is more often used for a classification process to distinguish between two different classes whereas the second uses Bayesian inference method to find connections between the nodes in regard to tablet attributes. We use the former for the classification of tablets manufactured from two size fractions of granules and the latter to establish whether there is any connection between the texture measures in equations 1-5 and the number of defects that are produced in a tablet due to tablet sticking). Bayesian networks graphical representations, which are used in both utilities, display the interactions found between the attributes (variables) where in our case they represent the texture measures applied to laser speckle image segments (independent variables) and the known physical feature of the tablet (i.e. granule size and the severity of defects, etc. as dependent variables). In $\mathrm{BN}$, each attribute is represented by a node which is connected to another node with a link only if there is substantial information flow [21] between these two attributes (Equation 7). Information flow refers to network characteristics by which two attributes communicate over their parent attribute. BNs are particularly used for modelling the joint probability distributions of variables. For example, if $\mathrm{A}=\left\{\mathrm{X}_{1}, \ldots \mathrm{X}_{\mathrm{n}}\right\}$ is a random variable which denotes patterns spanning the $n=N x M$ dimensional vector space, the joint probability distribution $\mathrm{P}=\left(\mathrm{X}_{1, \ldots} \mathrm{X}_{\mathrm{n}}\right)$ is then a product of all conditional probabilities and may be expressed by Equation 6.

$$
P(X)=\prod_{i} P\left(X_{i} \mid p a\left(X_{i}\right)\right)
$$

where $\mathrm{pa}\left(\mathrm{X}_{\mathrm{i}}\right)$ is the parent set of $\mathrm{X}_{\mathrm{i}}$ (e.g. the class node to decide on high/low density)

In order to accomplish the Bayesian classifier network construction, the PowerPredictor ${ }^{\mathrm{TM}}$ utility was used in the experiment for the classification of granule size. In Equation 7, $C$ is a set of nodes and $\mathbf{c}$ is an element (i.e. a vector) of $C$ ). If $I\left(A_{i}, A_{j} \mid C\right)$ is smaller than a certain threshold, $t$, then we can say that $A_{i}$ and $A_{j}$ are conditionally independent.

$$
I\left(A_{i}, A_{j} \mid C\right)=\sum_{a_{i}, a_{j}, c} P\left(a_{i}, a_{j}, c\right) \log \frac{P\left(a_{i}, a_{j} \mid c\right)}{P\left(a_{i} \mid c\right) P\left(a_{j} \mid c\right)}
$$


The Bayesian networks (BN) utility is a learning system. The learning process is implemented by a "training data set" which is selected from some portion (e.g. half) of the whole data set. The rest of the whole data set is then used as a "test set" to assess the reliability of the classification process (This is called the classification accuracy). Several parameters may be selected within the Bayesian utility tool (PowerPredictor ${ }^{\mathrm{TM})}$ in regard to data specification in order to obtain the best BN classification accuracy. These parameters include: (i) the threshold " $t$ " (which effects the number of nodes within the network), (ii) the discretization method (e.g. equal frequency, equal width, etc.), and (iii) the type of network (whether a single or a double classifier network). These software options are selected by trial and error using previous experience in training these networks.

\section{(d) Classification fusion (Multi-classifier system)}

Multi classifier systems have been studied in the past by several researchers [6][7][8]. It is generally understood that a combination of classifiers will outperform the best individual classifier. This idea has been proven analytically for some conditions by Tumer and Ghosh [9]. The MCS methods are introduced in detail by Ranawana and Palade [13]. In his study a specific MCS called "Average Vote" (shown by Equation 8 ) is the one which corresponds to our voting method. Here $C_{j i}(x)$ represents the vote that classifier $C_{j}$ performs with reference to input " $x$ " belonging to class $i$.

$$
\operatorname{MCS}_{\text {AVERAGEVOTE }}(x)=\arg \max _{i=1}^{C}\left(\frac{1}{R} \sum_{j=1}^{R} C_{j i}(x)\right)
$$

In our tablet characterization work, the optimization of classification results have been accomplished by using this method by merging three of different classification results where different laser light types, sampling windows, etc. are utilised for each individual classifier. The results are exhibited and further explained in the Results and Discussion section.

\section{Results and Discussion}

The data sets analysed comprise cases and attributes, where each case in the context of this work refers to an individual tablet and the attributes refer to the texture measures of different laser speckle image samplings on different laser speckle energy regions (shown in Figure 6). In the experiments in which the classification approach is taken (other than just an attribute connection test where Bayesian inference method is used to find the links between attributes), each data set is divided nearly equally to build the training and test where each one contains two different classes separately (e.g. high/low granule size, etc.). The classes are also distributed in the class node in an almost equal quantity.

\subsection{Tablet Sticking tests (microcrystalline cellulose and mefenamic acid)}

For the tests two group of tablets have been studied: one based on a wet granulated formulation of mefenamic acid and another based on the direct compression of microcrystalline cellulose (Avicel PH101). In the first test, IR and red laser speckle image samples of 20 mefenamic acid tablets (with progressively worsening of defects as they came off the tablet press) are taken with 10 different attributes corresponding to different texture measures with $3 \times 3$ and $5 \times 5$ pixel windows samplings. The results yielded by the Bayesian inference method (PowerConstructor ${ }^{\mathrm{TM}}$ ) (Figure 7) show that there is a direct link between one of the texture measures (i.e. the attribute Russ with $5 \times 5$ pixel operation window) and the time base for the sequence of tablets being produced by the compaction simulator. Given that the frequency and severity of defects also increases with time then there is a possibility that the Russ attribute is a marker for the tablet sticking process. If that is the case then we can assume that the test attribute of "time" refers to the progressive development of defects in the time domain, having hypothetical progressive defect values of

$\mathrm{t}=\{1,2,3,4,5 \ldots \mathrm{n}\}$. 


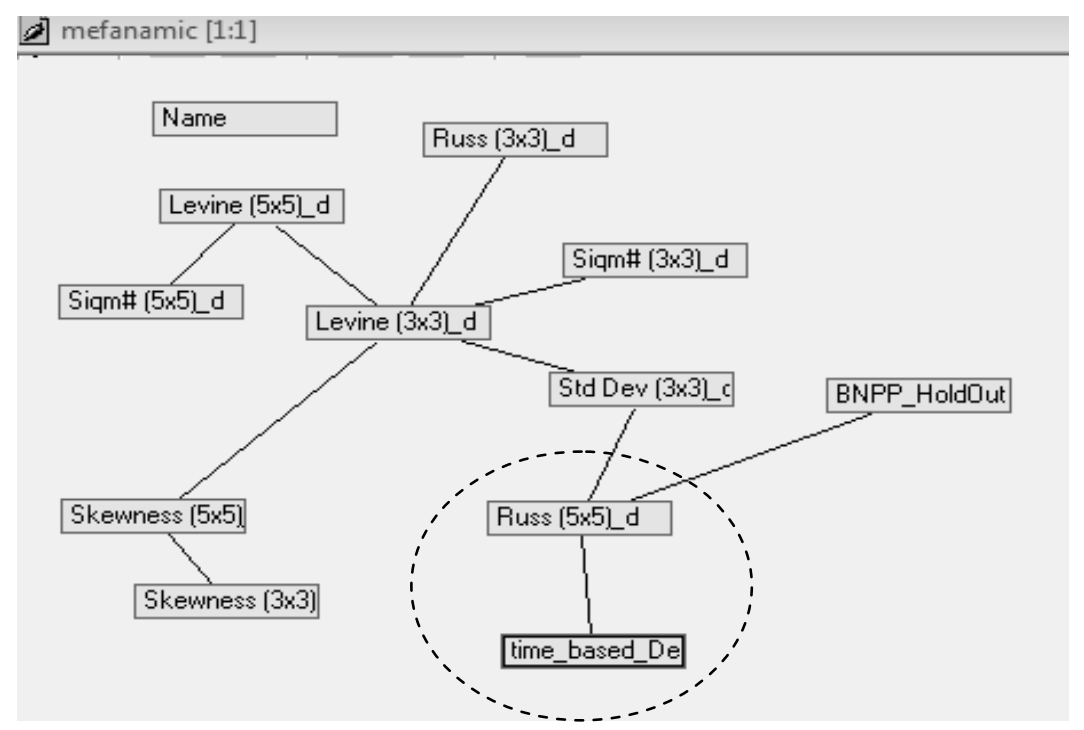

Figure 7. A direct link between the attribute Russ $(5 \times 5)$ and the attribute "time based defect is shown in this Bayesian network (mefenamic acid tablets). In the nodes the notations "d" refer to the discretisation applied to the continuous values within the data pre-processor.

In the second test, the microcrystalline cellulose (Avicel PH101) tablets that were prepared "without any defects" were photographed under laser illumination and a data set established, by laser speckle image samplings in same manner as in the first test on mefenamic acid tablets. In this case, the result (Figure 8) shows that there are no direct correlations between any of the texture parameters and the known test attribute of "time" variable (i.e. defect scale).

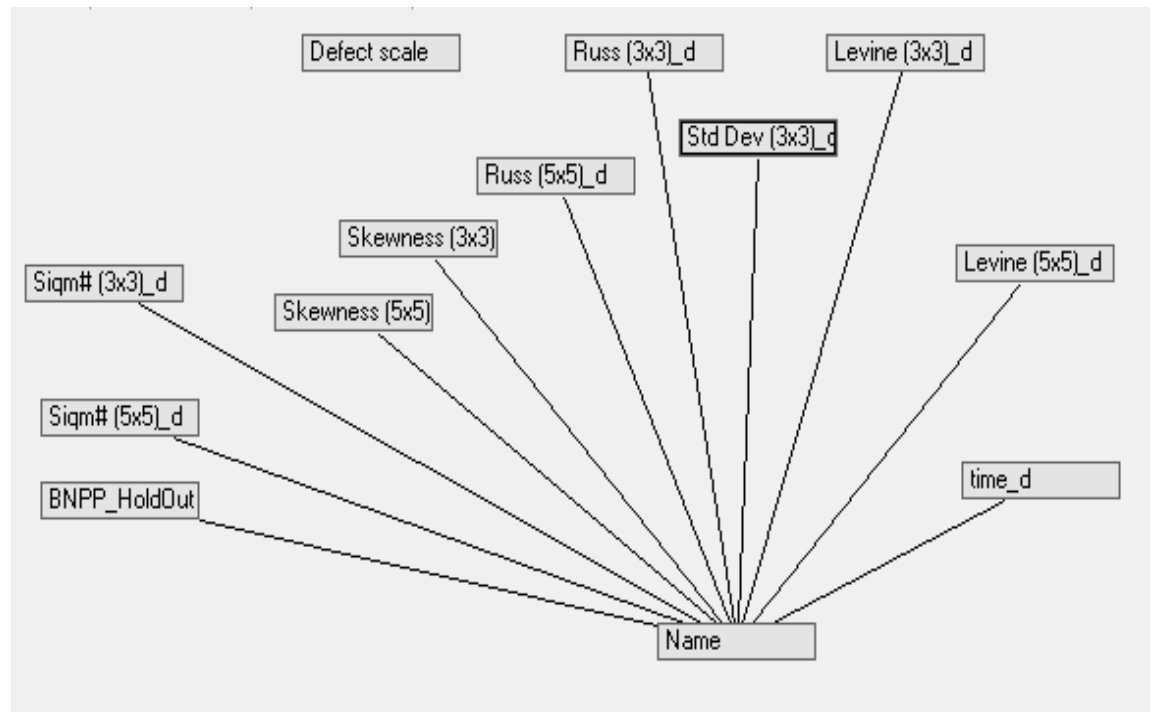

Figure 8. The $\mathrm{BN}$ shows that there are no direct links between any of the texture parameters and the known variable of time, where time is being taken as the "defect-scale" attribute, for the microcrystalline cellulose tablets) 


\subsection{Granule size tests (Classification \& Fusion)}

In this experiment a set of 18 wet granulated mefenamic acid tablets were used for the granule size classification process whose content is given in Table 1. Where the three different classification results are then fused to improve the ultimate classification result. Different classification processes are applied as follows: Classification 1 is made by using a red laser ( $80 \times 80$ pixel sampling), Classification 2 refers to IR laser ( $15 \times 15$ pixel sampling) and Class 3 refers to IR laser (30x30 pixel sampling) each for a different locations on the tablet surface. The classification results obtained are $75 \%, 75 \%$ and $62 \%$ respectively. In second stage of experiment, all classification processes are then fused (as seen in Table 5) by which the classification accuracy increases to $88 \%$.

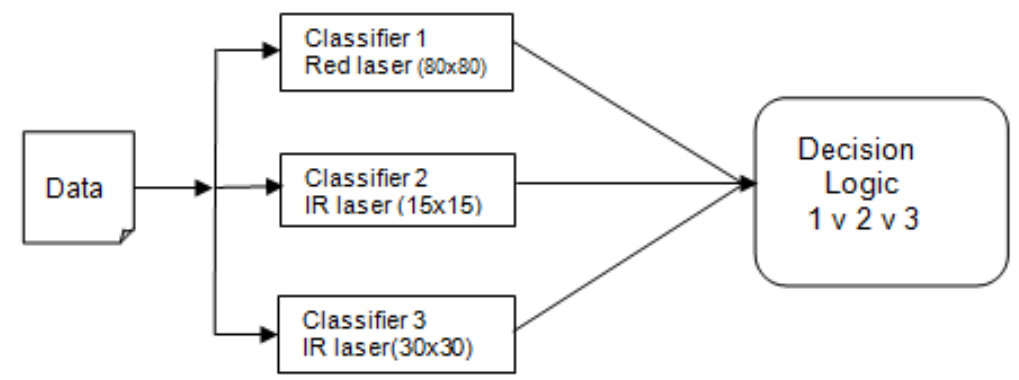

Figure 9. The basic representation of Multi-Classifier System (MCS) used for a two-category (separated by the average value) tablet classification process; where Class1 refers to red laser (80x80 pixel sampling), Class 2 refers to the IR laser (15x15) and Class3 refers to IR laser (30x30) each for different locations on the tablet surface. The fusion process is further explained in Table 5 . Decision logic votes between the results.

Table 5 -The fusion results of three different Bayesian classifiers (shown in Figure 9) whose classification results are $75 \%, 75 \%$ and $62 \%$ respectively. The fusion result is calculated as $88 \%$ for two category of tablet granule sizes (high $(\mathrm{H})=1 \mathrm{~mm}$, Low $(\mathrm{L})=0.25-0.50 \mathrm{~mm})$ for mefenamic acid.

\begin{tabular}{|c|c|c|c|c|c|c|c|c|c|c|}
\hline \multirow{2}{*}{$\begin{array}{l}\text { Test set } \\
\text { Tablet No }\end{array}$} & \multicolumn{3}{|c|}{ Classifier I } & \multicolumn{3}{|c|}{ Classifier II } & \multicolumn{3}{|c|}{ Classifier III } & \multirow{2}{*}{$\begin{array}{l}\text { Fusion } \\
\text { result }\end{array}$} \\
\hline & $\begin{array}{l}\text { Probe } \\
\text { out }\end{array}$ & $\begin{array}{l}\text { Probe } \\
\text { High dst. }\end{array}$ & $\begin{array}{l}\text { Probe } \\
\text { Low dst. }\end{array}$ & $\begin{array}{l}\text { Probe } \\
\text { out }\end{array}$ & $\begin{array}{l}\text { Probe } \\
\text { High dst. }\end{array}$ & $\begin{array}{l}\text { Probe } \\
\text { Low dst. }\end{array}$ & $\begin{array}{l}\text { Probe } \\
\text { out }\end{array}$ & $\begin{array}{l}\text { Probe } \\
\text { High dst. }\end{array}$ & $\begin{array}{l}\text { Probe } \\
\text { Low dst. }\end{array}$ & \\
\hline $1 \mathrm{H}$ & $\mathrm{H}$ & 0.74 & 0.25 & $\mathrm{H}$ & 0.75 & 0.25 & $\mathrm{~L}$ & 0.18 & 0.82 & $\mathrm{HH} \mathrm{L}=\mathrm{H}$ \\
\hline $2 \mathrm{H}$ & $\mathrm{H}$ & 0.74 & 0.25 & $\mathrm{H}$ & 0.75 & 0.25 & $\mathrm{~L}$ & 0.18 & 0.82 & $\mathrm{H} \mathrm{HL}=\mathrm{H}$ \\
\hline $3 \mathrm{H}$ & $\mathrm{H}$ & 0.74 & 0.25 & $\mathrm{~L}$ & 0.20 & 0.80 & $\mathrm{H}$ & 0.63 & 0.37 & $\mathrm{HLH}=\mathrm{H}$ \\
\hline $4 \mathrm{~L}$ & $\mathrm{H}$ & 0.74 & 0.25 & $\mathrm{H}$ & 0.75 & 0.25 & $\mathrm{~L}$ & 0.18 & 0.82 & $\mathrm{H} \mathrm{H} \mathrm{L}=\mathrm{H}$ \\
\hline $5 \quad \mathrm{~L}$ & $\mathrm{~L}$ & 0.20 & 0.80 & $\mathrm{H}$ & 0.75 & 0.25 & $\mathrm{~L}$ & 0.22 & 0.78 & $\mathrm{LH} \mathrm{L}=\mathrm{L}$ \\
\hline $6 \quad \mathrm{~L}$ & $\mathrm{~L}$ & 0.20 & 0.80 & $\mathrm{~L}$ & 0.20 & 0.80 & $\mathrm{~L}$ & 0.18 & 0.82 & $\mathrm{~L} \mathrm{~L} \mathrm{~L}=\mathrm{L}$ \\
\hline $7 \mathrm{~L}$ & $\mathrm{~L}$ & 0.20 & 0.80 & $\mathrm{~L}$ & 0.20 & 0.80 & $\mathrm{~L}$ & 0.37 & 0.63 & $\mathrm{~L} \mathrm{~L} \mathrm{~L}=\mathrm{L}$ \\
\hline $8 \mathrm{~L}$ & $\mathrm{H}$ & 0.74 & 0.25 & $\mathrm{~L}$ & 0.20 & 0.80 & $\mathrm{~L}$ & 0.37 & 0.63 & $\mathrm{HLL}=\mathrm{L}$ \\
\hline
\end{tabular}

Set of 18 mefenamic acid used for fusion process (Chapter 3.4) whose classification networks are shown in Figure 10 (top), and separate normal classification combination are shown in contingency tables 3 and 4 whose networks are also shown in Figure 10 (bottom). 

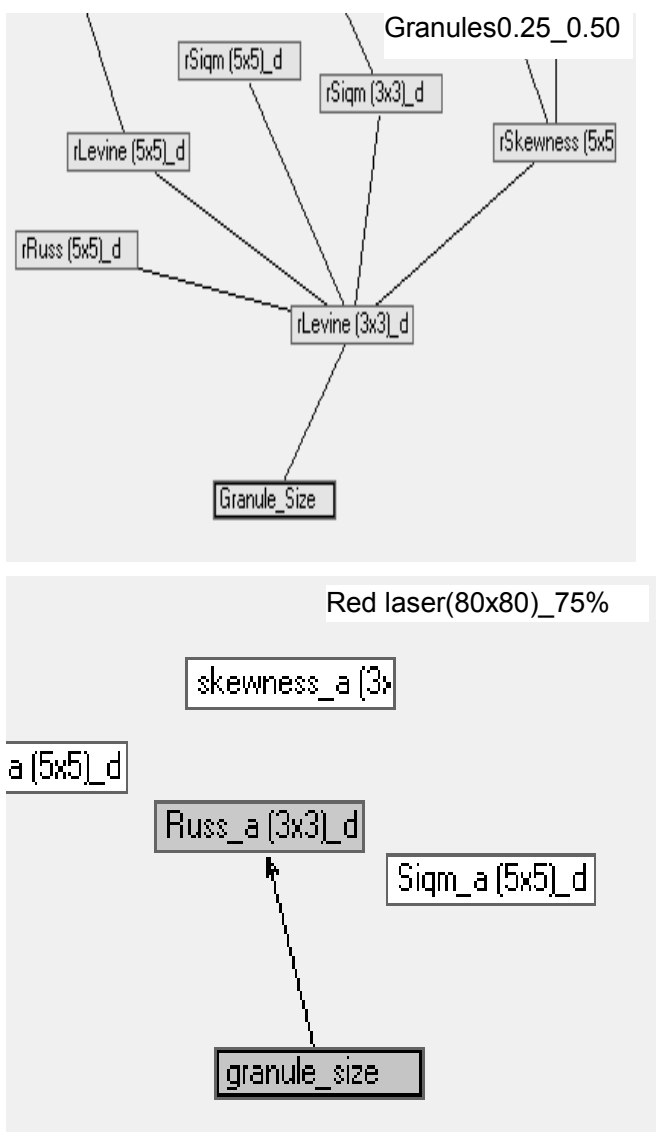

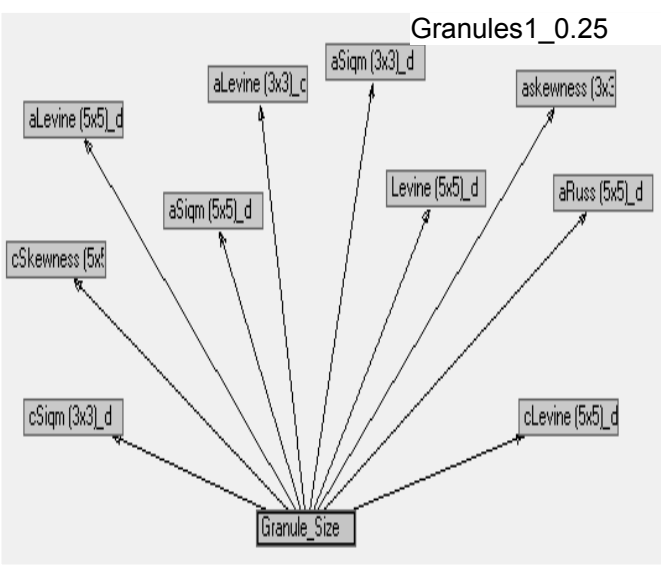

IR laser(15x15)_75\%

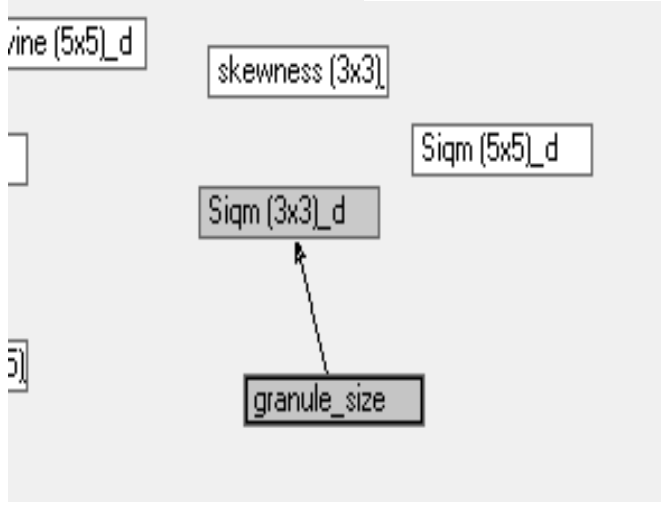

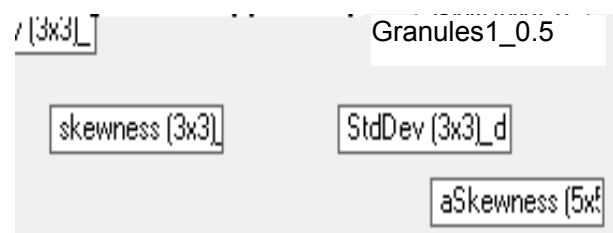

CRuss (3x3) d
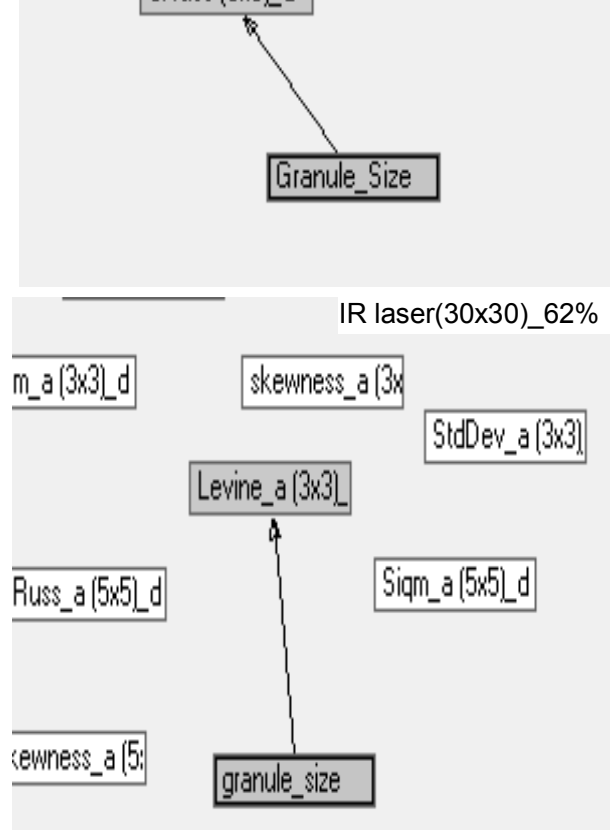

Figure 10. The networks used for fusion process where each one has different parameter configurations (1- red laser $(80 \mathrm{x} 80)$ with equal frequency discretization, $\mathrm{t}=0.1 ; 2$ - IR laser(15x15) with equal width discretization and ROC ; 3 - IR laser(30x30) with equal frequency discretization, $t=0.1$ ) respectively. The fusion process of three networks (with 75\%, 75\% and 62\% classification accuracy) yields the total classification accuracy of $88 \%$ (at the top). The networks used to classify the granulation sizes $0.25 \mathrm{v} 0.5 \mathrm{~mm}, 1 \mathrm{v} 0.25 \mathrm{~mm}$ and $1 \mathrm{v} 0.5 \mathrm{~mm}$ respectively. The classifications have been done in association with the contingency Table 4.

By the network fusion experiment it has been proven that different classifiers with different parameters, methods, materials, etc (used for same tablet set) can be merged to obtain optimum accuracy results. In this case the configurations of classifiers are as follows:

1 - Red laser (80x80 sampling windows) with equal frequency discretization, $\mathrm{t}=0.1$;

2- IR laser(15x15 sampling windows) with equal width discretization and ROC ;

3- IR laser(30x30 sampling windows) with equal frequency discretization, $t=0.1$ )

For each classifier a different laser type is used with the observation of different part of tablets, with different sampling windows. We have to note that the accuracy levels of BN results are not very precise and can vary with different minor conditions (e.g. different parameter selection, etc.). Hence 1-to-1 comparison between the tests may not be always possible. The other point is, the two cases (shown in Figure 10) are not 1-to-1 comparable because even though the same 18 mefenamic acid tablets are used in both experiments, their huge data set is utilized and for each case some parts of it selected automatically by BN, but selected manually by Multi-Classifier fusion methods for different classifications and also with different parameter options. 


\section{Conclusions}

This, our first investigation on the application of ILSC for the determination of tablet quality attributes and failure modes concentrated on two aspects: (i) the macroscale characteristics associated with the surface topography of the tablet and the impact of tablet sticking thereon, (ii) the microscale characteristics associated with the size of granules used in the preparation of tablets.

In the first part of the study, a set of mefenamic acid tablets with progressive defects was also used in tablet sticking tests where they showed that there is a direct link between one of the texture measures (i.e. the attribute Russ with $5 \times 5$ pixel operation window) and the time base for the sequence of tablets being produced by the compaction simulator. For the verification, a similar test was repeated with another set of Avicel tablets without defect, where the results did not show any indication of connection between the "defect" attribute and any of laser speckle texture measures which verified our tablet sticking hypothesis.

Given that, in the study, there was no consideration of what part of the defected tablet surface was being analysed (i.e. whether it was the defected or non-defected areas that were illuminated and sampled) then it may be the case that both the defected area and non-defected areas on the tablet within the defected group has some influence on the classification process (albeit the weighting from each might be different). The discriminating capability of the technique for surface defects may therefore be due, in part, to the fact that a potentially defected tablet (i.e. one that does not show any visible signs of defects but which might have otherwise developed into a defected tablet on another occasion) has characteristics that differ from a normal (non-defect) tablet. This raises the prospect that the technique could be used in the early prediction of sticking on long runs times, on production presses at high production speeds, where heating of the press could soften the tablet components and increase the probability of sticking.

In the second part of the study, sets of mefenamic acid tablets were produced from three different granules size. The relatively high classification accuracy result of $88 \%$ for granule size of mefenamic acid is yielded by the fusion of three separate classification processes. The t-tests results of densities for the two granulation size categories $(1 \mathrm{~mm} \mathrm{v}$ $0.25-0.5 \mathrm{~mm})$ and $(0.25 \mathrm{v} 0.5 \mathrm{~mm})$ confirm that their differentiation by t-tests coincide with the classification results of same granulation size categories.

By all experiments, the most appropriate use of laser wavelength for each test is also concluded by the automated selection of Bayesian classifier or inference algorithm, for the tests on mefenamic acid tablets) where IR and red lasers were particularly found suitable for reaching higher classification accuracy than a single laser wavelength. It was also found that the comparatively higher energy $(5 \mathrm{~mW})$ IR laser light generates different speckle effect by interacting with deeper sub-surface of the tablets than the lower energy $(3 \mathrm{~mW})$ red laser where the unification of both kinds of speckle images provides higher discrimination power to distinguish between the tablet sub component variations, such as granule size.

The overall conclusion is that with certain controls (i.e. tests done in the same lab under similar conditions) then the proposed system has the potential to be used for industrial tablet inspection purposes on a real-time basis. This is on the basis that any potential problem originated from a conditional change in characteristics of the experimental set up may be eliminated by an initial calibration process at the beginning of each inspection session by using a set of test materials whose laser speckle parameters are already known. Further improvements could be achieved in the experimental set up by using higher power lasers and a broader range of laser sources (wavelengths) along with auxiliary optical components, particularly a filtering mechanism which prevents high power laser light reflection from the tablet surface. In order to improve on the image analysis methods then other classification approaches such as Support Vector Machines (SVM) will also be used in future studies, in which a higher number of classes (e.g. five types of granules) could be processed simultaneously instead of the two classes, as was the case in this short study. 


\section{References}

[1] Orun, A.B. “ intelligent Laser Speckle Classification “ Wikipedia Encyclopaedia, http://en.wikipedia.org/wiki/Intelligent_Laser_Speckle_classification.

[2] Orun, A.B, E. Goodyer, H.Seker, G. Smith, V.Uslan And D. Chauhan, "Optimized parametric skin modelling for diagnosis of skin abnormalities by combining light back-scatter and laser speckle imaging", Skin Research and Technology, 2014; 1-13. doi:10.1111/srt.12142.

[3] Garcia-Munoz, S. and D. S. Gierer. Coating uniformity assessment for colored immediate release tablets using multivariate image analysis. International Journal of Pharmaceutics 395 (2010) 104-113.

[4] Orun, A.B and A.Alkis.2003. Material identification by surface reflection analysis in combination with bundle adjustment technique, Pattern Recognition Letters,24(2003),pp.1589-1598.

[5] Orun, A.B and Aydin, N. "Variable optimisation of medical image data by the learning Bayesian Network reasoning", Proceedings of the $32^{\text {nd }}$ Annual International Conference of the IEEE Engineering in Medicine and Biology Society (EMBC'10), Buenos Aires, Argentina, 1st - 4th September, 2010.

[6] Wozniak, M., M. Grana and E.Corchado. A survey of multiple classifier systems as hybrid systems. Information Fusion, Vol. 16, March 2014, Pages 3-17.

[7] T. Ho, J.J. Hull, S. Srihari Decision combination in multiple classifier systems. IEEE Transactions on Pattern Analysis and Machine Intelligence, 16 (1) (1994), pp. 66-75.

[8] Clemen, R. Combining forecasts: a review and annotated bibliography. International Journal of Forecasting, 5 (4) (1989), pp. 559-583.

[9] Tumer, K. and J. Ghosh. Analysis of decision boundaries in linearly combined neural classifiers. Pattern Recognition, 29 (2) (1996), pp. 341-348.

[10] Dainty, C. , Laser Speckle and Related Phenomena, 1984, Sprinter Verlag, ISBN 0-387-13169-8.

[11] Briers, J.D and S. Webster, “ Laser speckle contrast analysis (LASCA) : a non scanning, full-field technique for monitoring capillary blood flow", Journal of Biomedical Optics, Vol. 1, No.2., April 1996.

[12] Stephens, J.D., M.V. Lakshmaiah, B.R. Kowalczyk, B.C. Hancock and C. Cetinkaya. Wireless transmission of ultrasonic waveforms for monitoring drug tablet properties and defects. International Journal of Pharmaceutics 442 (2013) 35- 41.

[13] Ranawana, R. and V. Palade . Multi-Classifier Systems - A Review and roadmap for developers, University of Oxford Computing Lab. ,Oxford. December 13, 2005.

[14] Feng, L., W. Xinxin, C. Yifeng, Y. Yongjian, Y. Yinjia and D. Gengli. A novel identification system for counterfeit drugs based on portable Raman spectroscopy. Chemo metrics and Intelligent Laboratory Systems 127 (2013) 63-69.

[15] Torrance, K and E. Sparrow. Theory of off-specular reflection from roughened surfaces, J. of Optical Soc. Am. A. 57, (1967), 1105-1114.

[16] Wolff, L. A diffuse reflectance model for smooth dielectrics, J. of Optical Soc. Am. A. 11, (1994), 2956-2968.

[17] Tominaga, S. Surface identification using the dichromatic reflection model. IEEE Trans. Pattern Analysis \& Machine Intelligence , 1991, 13 (7).

[18] Nayar, S.K. and Oren, M. Visual appearances of matte surfaces. Science 26 (February 1995),1153 - 1156. 
[19] Phillips, D., 1995. Image processing in C, Part 15: Basic texture operations, C/C++ Users Journal, November.

[20] Kihm, K.D. Laser speckle photography technique applied for heat and mass transfer problems, Advances In Heat Transfer, Vol. 30 (1997), pp. 255-311.

[21] http://wiki.cs.byu.edu/cs-677sp2010/undirected-models

[22] Hernandeza, S.E., P. Pallavi, G. Keyvan, Y. Wang, N. Velez,G. Callegari, A. Cuitino, B. Michniak-Kohn, F. J. Muzzio,R. J. Romanach. Prediction of dissolution profiles by non-destructive near infrared spectroscopy in tablets subjected to different levels of strain, Journal of Pharmaceutical and Biomedical Analysis, 117 (2016) $568-576$.

[23] Krystan, R., C. Davies and K. Kelly. Tablet sticking: Using a 'compression toolbox' to assess multiple tooling coatings options. Powder Technology, Volume 285, November 2015, Pages 103-109.

[24] Bawuah, P., A.P. Mendia, P. Silfsten, P. Pääkkönen,, T. Ervasti, , J. Ketolainen, J. A. Zeitler and K. Peiponen, Detection of porosity of pharmaceutical compacts by terahertz radiation transmission and light reflection measurement techniques, International Journal of Pharmaceutics 465 (2014) 70-76

[25] Peiponen, K, P. Bawuah, M. Chakraborty, M. Juuti, J. A. Zeitler, J. Ketolainen. Estimation of Young's modulus of pharmaceutical tablet obtained by terahertz time-delay measurement. International Journal of Pharmaceutics 489 (2015) 100-105

[26] Silvennoinen, R, V. Hyvarinen, P. Raatikainen, K. Peiponen, Dynamic laser speckle pattern in monitoring of local deformation of tablet surface after compression. International Journal of Pharmaceutics 199 (2000) 205-208

[27] Anders Axelsson, A., M. Marucci. The use of holographic interferometry and electron speckle pattern interferometry for diffusion measurement in biochemical and pharmaceutical engineering applications. Optics and Lasers in Engineering 46 (2008) 865-876 